\title{
Sand Fly Evolution and its Relationship to Leishmania Transmission
}

\author{
PD Ready \\ Molecular Systematics Laboratory, Department of Entomology, The Natural History Museum, \\ London SW7 5BD, UK
}

The evolutionary relationships of sand flies and Leishmania are discussed in this report, which draws distinctions between co-association, co-evolution and co-speciation (or co-cladogenesis). Examples focus on Phlebotomus vectors of Le. infantum and Le. major in the Mediterranean subregion.

Key words: co-evolution - Phlebotomus - Leishmania

There is a noticeable association between certain Old World species or species complexes of Leishmania and particular subgenera of Phlebotomus, as characterized by isoenzymes and morphology respectively. The term "evolutionary fit" has been used (Killick-Kendrick 1985), but Esseghir et al. (2000) concluded that clear distinctions have not always been drawn between co-association (with the transmission cycle having a distinctive "landscape epidemiology"), co-evolution (e.g. between molecules of the sand fly midgut and the parasite external surface) and vector-parasite cospeciation (or co-cladogenesis).

The evolutionary relationships between sand flies and Leishmania would seem to have implications for leishmaniasis control or intervention. For example, putative vectors in previously unexplored foci can usually be targeted simply by their taxonomic relationship to known vectors. In the Mediterranean subregion, western Asia and sub-Saharan Africa all the firmly incriminated vectors of Le. major (but not some of its suspected vectors) are classified in the subgenus Phlebotomus and, similarly, most of the firmly incriminated vectors of Le. tropica and Le. infantum are placed in the subgenera Paraphlebotomus and Larroussius, respectively (Killick-Kendrick 1990). These might be longstanding evolutionary relationships, in which taxa of parasites and vectors are locked together by unique behavioural or molecular phenotypes of epidemiological importance. Thus, only P. (Phlebotomus) papatasi would seem to have the midgut molecules to which the highly substituted side-chains of the lipophosphoglycans (LPGs) of Le. major can bind (Pimenta et al. 1994). However, too few parasite-vec-

Fax: +44-20-7942.5229. E-mail: P.Ready@nhm.ac.uk Received 13 April 2000

Accepted 15 May 2000 tor combinations have been experimentally investigated for one to conclude that ligand-receptor coevolution in the midgut is the key to the association between Le. major and the subgenus Phlebotomus. The determinants of the arid-habitat associations of the vectors and the gerbil reservoir hosts might also be critical. So far, based on cytochrome b sequences of sand fly mitochondrial DNA (mtDNA), there is no evidence for co-cladogenesis between the four species in the subgenus Phlebotomus and zymodemes of Le. major (Esseghir et al. 1997). The same zymodemes have been isolated from $P$. duboscqi south of the Sahara and from P. papatasi in the Mediterranean subregion. The geographical populations of $P$. papatasi are separated by small genetic distances in a way consistent with a recent radiation from the eastern Mediterranean Basin to north Africa, southern Europe and western Asia (Esseghir et al. 1997), but there is no clear evidence for a similar radiation of Le. major strains.

There has been speculation about the evolutionary relationships between Phlebotomus of the subgenus Larroussius and Le. infantum, perhaps because some strains of this parasite have been associated with cutaneous rather than visceral human disease. The mode of speciation of Mediterranean Larroussius has recently been inferred from comparative sequence analyses not only of mtDNA but also of a nuclear gene, elongation factor alpha (Esseghir et al. 2000). The molecular phylogenies were congruent basally, where their clades matched species complexes defined by a few genitalic characters of each sex. For the most derived species complex (P. perniciosus), however, there was strong evidence for reticulate evolution. The molecular phylogenies were incongruent and mtDNA marker distribution was consistent with introgressive hybridizations between species whose current ranges overlap or abut. Reticulate evolution in the $P$. perniciosus complex (Pesson et al. 1999, Ready \& Pesson 1999) makes it difficult to detect co-cladogenesis with Leishmania. 
Esseghir et al. (2000) considered not only the molecular phylogenies but also the ecological niches of the Larroussius species and the historical biogeography and palaeoecology of the Mediterranean subregion. They concluded that there had been sequential speciation (a "taxon pulse") promoted by increasing aridification in the Pliocene, later than previously proposed but too early for Pleistocene Ice-age refugia to have played a part. Speciation would have occurred before the proposed divergence of Le. donovani and its sister species Le. infantum, and this helped to rule out any strict vector-parasite co-speciation or co-cladogenesis with strains.

The conclusions of Esseghir et al. (2000) depended on a molecular clock for mtDNA. The sand fly fossil record is poor (Lewis 1982), and so proposals concerning the speciation of sand flies should be supported by molecular-clock datings allied to resolved (molecular) phylogenies, rather than general hypotheses about vicariance and tectonic activities. The dating of the divergence of $L e$. donovani and Le. infantum was based on an isoenzyme clock (Moreno et al. 1986), and this should be compared with DNA clocks.

The absence of any unequivocal evidence for strict co-cladogenesis in the species-level examples considered above emphasizes the need for more precise biological explanations of the strong associations between some groups of sand flies and Leishmania. Inferences based on phylogenetic relationships can be used to formulate interesting evolutionary hypotheses, but these should be tested experimentally. For example, is the structure of the LPG of Le. major a critical factor for explaining the ability of all members of the subgenus Phlebotomus to transmit this parasite, or is P. papatasi (the only species investigated experimentally) unique in this respect?

The evolution of the molecules involved in vector-parasite specificity may not always be directly linked to the speciation (or cladogenesis) of their hosts, especially if there is gene introgression. In this respect, it is becoming clear that interspecific hybridization and gene introgression can be expected among recently evolved sand fly morphospecies, in the American genus Lutzomyia (Marcondes et al. 1997) as well as in the Phlebotomus subgenera Larroussius and Phlebotomus, for which there is experimental evidence of interbreeding (see Esseghir et al. 1997).

Co-cladogenesis, and its epidemiological significance, should be more easily discernible among higher taxonomic groups, after the completion of "lineage sorting" aided by reproductive barriers. Molecular data are helping to define some of these higher taxonomic groups. For example, based on small subunit nuclear ribosomal RNA gene sequences, it is clear that most (if not all) Phlebotomus vectors of parasites in the Le. donovani complex belong to one monophyletic group, which includes sand flies in the subgenera Euphlebotomus and Adlerius in addition to Larroussius (Aransay et al. 2000).

\section{ACKNOWLEDGEMENTS}

To Ana Aransay, Selma Esseghir, Riadh Ben-Ismail, Bob and Mireille Killick-Kendrick, Bernard Pesson, Jo Testa and many other colleagues for their input during the course of joint projects.

\section{REFERENCES}

Aransay AM, Scoulica E, Tselentis Y, Ready PD 2000. Phylogenetic relationships of phlebotomine sandflies inferred from small subunit nuclear ribosomal DNA. Insect Mol Biol 9: 157-168.

Esseghir S, Ready PD, Ben-Ismail R 2000. Speciation of Phlebotomus sandflies of the subgenus Larroussius coincided with the late Miocene-Pliocene aridification of the Mediterranean subregion. Biol J Linn Soc 69: in press.

Esseghir S, Ready PD, Killick-Kendrick R, Ben-Ismail R 1997. Mitochondrial haplotypes and phylogeography of Phlebotomus vectors of Leishmania major. Insect Mol Biol 6: 211-225.

Killick-Kendrick R 1985. Some epidemiological consequences of the evolutionary fit between leishmaniae and their phlebotomine vectors. Bull Soc Path Exot 78: 747-755.

Killick-Kendrick R 1990. Phlebotomine vectors of the leishmaniases: a review. Med Vet Entomol 4: 1-24.

Lewis DJ 1982. A taxonomic review of the genus Phlebotomus (Diptera: Psychodidae). Bull Br Mus Nat Hist (Ent) 45: 121-209.

Marcondes CB, Day JD, Ready PD 1997. Introgression between Lutzomyia intermedia and both $\mathrm{Lu}$. neivai and $\mathrm{Lu}$. whitmani, and their roles as vectors of Leishmania braziliensis. Trans $R$ Soc Trop Med Hyg 91: 725-726.

Moreno G, Rioux JA, Lanotte G, Pratlong F, Serres E 1986. Le complexe Leishmania donovani s.l. In JA Rioux, Leishmania. Taxonomie et Phylogénèse. Applications Éco-épidémiologiques, Colloques Internationaux CNRS/INSERM, Montpellier, France.

Pesson B, Ready PD, Benabdennbi I, Esseghir S, CadiSoussi M, Morillas-Marquez F 1999. Reticulate evolution of $P$. perniciosus and $P$. longicuspis. International Symposium on Phlebotomine Sandflies III, Montpellier, France.

Pimenta PFP, Saraiva EMB, Rowton E, Modi GB, Garraway LA, Beverley SM, Turco SJ, Sacks DL 1994. Evidence that the vectorial competence of phlebotomine sand flies for different species of Leishmania is controlled by structural polymorphisms in the surface lipophosphoglycan. Proc Natl Acad Sci USA 91: 9155-9159.

Ready PD, Pesson B 1999. Hybridization, introgression and distribution of vectorial traits. International Symposium on Phlebotomine Sandflies III, Montpellier, France. 